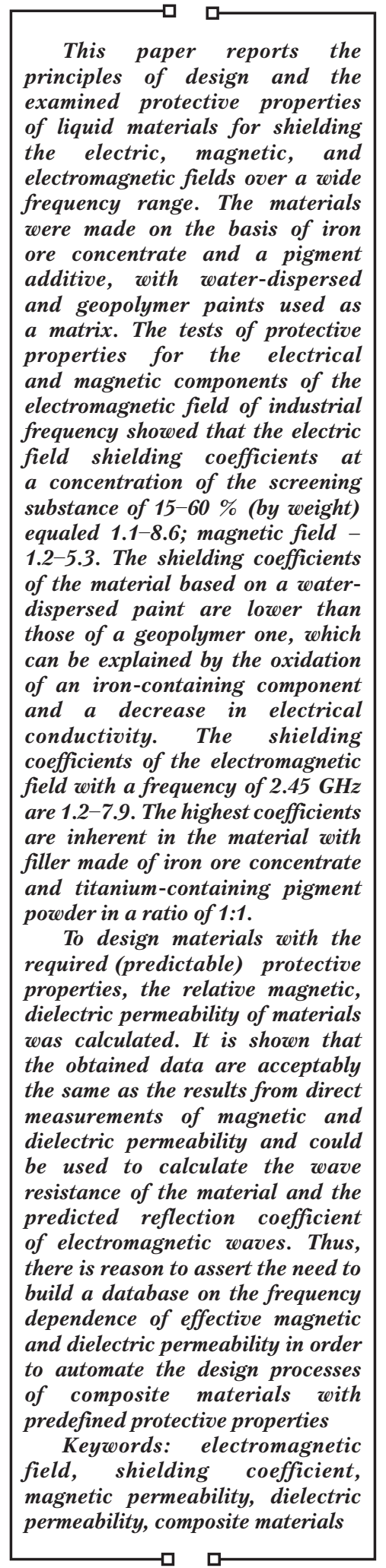

\title{
DESIGN OF LIQUID COMPOSITE MATERIALS FOR SHIELDING ELECTROMAGNETIC FIELDS
}

\author{
Valentyn GIyva \\ Doctor of Technical Sciences, Professor \\ Department of Civil and Industrial Safety* \\ Volodym yr Bakharev \\ Doctor of Technical Science, Associate Professor, \\ Dean Faculty of Natural Sciences \\ Kremenchuk Mykhailo Ostrohradskyi National University \\ Pershotravneva str., 20, Kremenchuk, Ukraine, 39600 \\ Natalia Kasatkina \\ Doctor of Technical Science, Head of Department \\ Department of Doctoral and Postgraduate Studies \\ National University of Food Technologies \\ Volodymyrska str., 68, Kyiv, Ukraine, 01601 \\ O leg Levchenko \\ Doctor of Technical Sciences, Professor, Head of Department \\ Department of Labor protection, Industrial and Civil Safety** \\ Larysa Levchenko \\ Doctor of Technical Sciences, Associate Professor \\ Department of Automation of Projection of Power Processes and Systems** \\ Natai a Burdeina \\ $\mathrm{PhD}$, Associate Professor*** \\ Sergi i G uz i i \\ $\mathrm{PhD}$, Senior Researcher \\ Department of Fire Prevention in Settlements \\ National University of Civil Defence of Ukraine \\ Chernyshevska str., 94, Kharkiv, Ukraine, 61023 \\ O lena Panova \\ PhD, Associate Professor, Head of Department*** \\ Oks a n a $\mathbf{~ y k h e n k o ~}$ \\ Corresponding author \\ $\mathrm{PhD}$, Associate Professor \\ Department of Ecology* \\ E-mail: okstih@ua.fm \\ Yana Biruk \\ Assistant*** \\ *National Aviation University \\ Liubomyra Huzara ave., 1, Kyiv, Ukraine, 03058 \\ $* *$ National Technical University of Ukraine \\ "Igor Sikorsky Kyiv Polytechnic Institute" \\ Peremohy ave., 37, Kyiv, Ukraine, 03056 \\ ***Department of Physics \\ Kyiv National University of Construction and Architecture \\ Povitroflotsky ave., 31, Kyiv, Ukraine, 03037
}

Received date: 01.04 .2021 Accepted date: 13.05 .2021 Published date: 18.06 .2021
How to Cite: Glyva, V., Bakharev, V., Kasatkina, N., Levchenko, O., Levchenko, L., Burdeina, N., Guzii, S., Panova, O., Tykhenko, O., Biruk, Y. (2021). Design of liquid composite materials for screening electromagnetic fields. Eastern-European Journal of Enterprise Technologies, 3 (6 (111)), 25-31. doi: https://doi.org/10.15587/1729-4061.2021.231479

\section{Introduction}

World scientific and technical development has led to the fact that there is a steady increase in the electromagnetic load on the industrial environment and the environment as a whole. This is due to an increase in the number and density of the location of electrical and electronic equipment in production facilities, an increase in the load on power 
electrical networks, and the development of wireless communication means. The transition to $5 \mathrm{G}$ mobile communication standards with the use of extremely high-frequency electromagnetic radiation requires an increase in the number and density of the location of base stations to increase the maximum permissible levels of radiation. Therefore, much attention is paid to the study of this problem. Back in 1995, the World Health Organization (WHO) officially introduced the term "global electromagnetic pollution". WHO included the problem of electromagnetic pollution in the list of humanity's priority problems, and, in 1996, WHO created an international project to identify potential health risks associated with technologies that generate electromagnetic fields. WHO requires maximum reduction of field levels to a clear clarification of the limits of permissible exposure to electromagnetic fields, depending on frequencies and amplitudes. Thus, the principle of maximum reduction under industrial conditions covers electromagnetic fields until reliable and unambiguous data on the maximum permissible levels are received. That is, this physical factor is covered by the principle of ALAR (as low as reasonably - as low as it is reasonably achievable). This principle is used even in developed countries in the variant of ALARP (as low as reasonably practicable), which implies establishing a reasonable level of danger as low as it could be achieved practically taking into consideration social factors. This principle requires the employer to create conditions for maximum reduction of the risks of negative changes in the health of employees under the influence of the electromagnetic factor.

At the same time, medical studies indicate the possibility of adverse health shifts under the influence of electromagnetic fields of low intensities [1, 2]. This requires justification and development of appropriate measures and means for protecting people under industrial and household conditions. The most effective method of reducing the levels of electromagnetic fields of the wide frequency range is shielding. Under the conditions of significant contribution to the electromagnetic situation of electromagnetic fields of ultra-high and higher frequencies, the use of traditional metal shielding surfaces is not always acceptable due to high reflection coefficients. That predetermines the possible direction of reflected radiation in an undesirable direction. The most acceptable are composite screening materials, whose main advantage is the controllability of shielding coefficients by changing the concentration of the screening substance in the matrix. Disadvantages of the use of composite materials are the complexity of manufacturing technologies and high cost. However, most of them have large thicknesses, which makes it difficult to use them for facing surfaces of large areas, as well as high price. Therefore, it is important to design highly efficient, broadband, and easy-to-use composite materials. To solve the task of shielding individual premises, parts of buildings, it is advisable to explore the possibility of production and application of shielding materials on liquid media. Such materials are more practical when applied to the surface of any shape with a minimum amount of waste and adjusting the thickness of the protective layer.

\section{Literature review and problem statement}

Much attention is paid to the development and study of the protective properties of composite materials for shielding electromagnetic fields. Most of them are metal-polymer structures. Study [3] identified the protective properties of a material made of polymer matrix and a metal filler made of ferrite of micro size. Recently, a significant part of studies addresses the development of compositions based on metal and metal-containing nanostructures. Article [4] describes a protective material based on nanocarbon with a matrix of complex hydrocarbon polymer. In [5], the matrix is made of common polypropylene, and filler - nanoparticles such as ferrite and graphene. These materials have higher, compared to composite materials with microparticles, screening coefficients. However, the issues related to manufacturing technologies and high cost remained unresolved. Such material is difficult to clad the surfaces of a complex configuration. In many cases, there are problems with the adhesion of the polymer. More acceptable are liquid protective materials that could be applied on surfaces of any shape with the desired thickness. This approach was used in work [6], which shows the results of studies of the composition of the type of putty containing tungsten (70\%), nickel (18.6\%), and carbon $(11.4 \%)$. However, such material is designed to protect against ionizing radiation and is designed to replace lead. Study [7] suggested a material based on carbon and aerogel. However, at acceptable protective properties, the mechanical properties of coatings are unsatisfactory. The technological advance reported in [8] and the above screening coefficients indicate the possibility of manufacturing protective putty and plasters by introducing magnetic and dielectric filler into them. However, their thickness is up to $10 \mathrm{~mm}$, and the coating is two-layer. This complicates practical application and increases the cost of work. Detailed studies $[9,10]$ have shown that the use of finely dispersed graphite and graphitized soot as a polymer matrix filler provides high screening coefficients over wide bands of frequencies. However, these fillers (especially graphitized soot) are produced in small volumes and have a high cost. The polymer matrix does not always provide ease of facing surfaces of complex configuration. Paper [11] reports the results of studying the rheological and adsorption properties of water-dispersed and synthetic paints based on geopolymers. All this suggests that such paints could be used as the base of metal-containing compositions for shielding electromagnetic fields. It is also advisable to conduct research into their screening coefficients of electrical, magnetic, and electromagnetic fields of the most common frequencies.

Our review allows us to conclude that most composite materials are designed for a narrow purpose. By functionality and cost, they are not adapted for facing surfaces of large areas and complex configurations. No fact-based data on the protective properties of liquid materials based on standard paint and varnishes are available. Known analytical ratios for determining the magnetic and electrophysical mixtures are not adapted to the forecasting of magnetic, electrophysical properties of liquid composite materials. Solving these problems would make it possible to devise the general principles of designing broadband electromagnetic screens on liquid media.

\section{The aim and objectives of the study}

The aim of this study is to identify the protective properties of materials based on paints with different content of a metal substance, to provide an estimation apparatus for the development of electromagnetic screens with controlled protective properties. 
To accomplish the aim, the following tasks have been set:

- to investigate the shielding coefficients by liquid composite materials of electrical, magnetic fields of industrial frequency and ultra-high frequency electromagnetic fields;

- to devise the principles of designing protective materials by predicting the magnetic and electrophysical properties of materials at different ratios of component content.

\section{Materials and methods to study liquid composite materials for shielding electromagnetic fields}

For the convenience of applying a protective material on the surface, ready-made paints of two types were chosen as a matrix. The first is the acrylic water-dispersion paint VD-AK-22W (Belarus) $\left(\rho=1.03 \mathrm{~g} / \mathrm{cm}^{3}\right)$, the second is the geopolymer paint MK 3/18-9.20 (Ukraine) $\left(\rho=1.15 \mathrm{~g} / \mathrm{cm}^{3}\right)$.

Fine iron ore concentrate obtained by flotation at Poltava Mining and Processing Plant (Ukraine) with a Fe content of $68-72 \% ; \mathrm{Fe}_{3} \mathrm{O}_{4}, 20-22 \%\left(\rho=6.24 \mathrm{~g} / \mathrm{cm}^{3}\right)$, was used as a screen filler. Also, the pigment mixture GreyX produced by "Ukrainian Mineral Company", Kyiv (Ukraine), with the content of $\mathrm{Al}_{2} \mathrm{O}_{3}$ of $50 \%$; $\mathrm{TiO}_{2}, 25 \% ; \mathrm{Fe}_{2} \mathrm{O}_{3}, 10 \%$, was used as a filler.

It is known [12] that the effectiveness of shielding depends on the dispersion of the filler, so the granulometric analysis of mixtures by the standard method of sedimentation was performed. The average particle size of iron ore concentrate after grinding was $22-23 \mu \mathrm{m}$, GrayX mixture $-8-9 \mu \mathrm{m}$. Shielding mixtures were made by mixing the paint with the desired amount of filler in the laboratory dissolver. Three types of screen protective material were manufactured:

- a water-dispersion paint with the addition of iron ore concentrate in weights of $15,30,45,60 \%$ (material No. 1);

- a geopolymer paint with the addition of iron ore concentrate in weights of $15,30,45,60 \%$ (material No. 2);

- a geopolymer paint with the addition of a mixture of iron ore concentrate and GreyX in a ratio of 1:1 in weights of 15, 30, 45, 60 \% (material No. 3).

All the materials were applied to the surface. After drying, the thickness of the protective layer was $0.22-0.25$.

The intensity measurement of the electrical and magnetic components of the electromagnetic field of industrial frequency was carried out by the calibrated meter BE-meter, modifications "AT-004" and "50 Hz", with a control unit "HTM-terminal”(Russian Federation), according to the operating instructions. The limit of permissible relative error of measurements of mean square values of electric and magnetic fields intensities was $15 \%$. To determine the shielding coefficients of electromagnetic fields of ultrahigh frequencies, we used the calibrated energy flux density meter PZ-31 (Russian Federation) according to the operating instructions. The maximum basic error of measurement did not exceed $2.7 \mathrm{~dB}$.

The shielding coefficients were measured for geometrically closed screens of cubic shape, measuring $0.2 \times 0.2 \times 0.2 \mathrm{~m}$, made of plasterboard, and covered with a protective mixture.

The measuring antenna was placed inside the shielding structure through a technological hole, which was tightly closed during the measurement process.

\section{Investigating the protective properties of liquid composite materials in terms of shielding of electromagnetic fields}

5. 1. Studying the shielding coefficients of electric, magnetic, and electromagnetic fields of a wide frequency range

The results of measuring the shielding coefficients of the electric field of the industrial frequency with an intensity of $178-180 \mathrm{~V} / \mathrm{m}$ by the developed materials are given in Table 1 and shown in Fig. 1.

The sample of material No. 1 - a water-dispersion paint with the addition of iron ore concentrate in weights of 15 , $30,45,60 \%$

The sample of material No. 2 - a geopolymer paint with the addition of iron ore concentrate in weights of $15,30,45,60 \%$.

The sample of material No. 3 - a geopolymer paint with the addition of a mixture of iron ore concentrate and GreyX in a ratio of $1: 1$ in weights of $15,30,45,60 \%$.

Table 1

Shielding coefficients $K_{S}$ of the electric field of industrial frequency*

\begin{tabular}{|c|c|c|c|c|}
\hline \multirow{2}{*}{$\begin{array}{c}\text { Sample of } \\
\text { material }\end{array}$} & \multicolumn{4}{|c|}{$K_{S}$} \\
\cline { 2 - 5 } & $15 \%$ & $30 \%$ & $45 \%$ & $60 \%$ \\
\hline No. 1 & $1.1-1.2$ & $1.3-1.4$ & $1.6-1.7$ & $2.8-2.9$ \\
\hline No. 2 & $1.1-1.2$ & $1.6-1.7$ & $2.9-3.0$ & $5.2-5.3$ \\
\hline No. 3 & $1.3-1.4$ & $1.8-1.9$ & $4.2-4.3$ & $8.5-8.6$ \\
\hline
\end{tabular}

Note: *Shielding factor $K_{S}=E_{f} / E_{S}$, where $E_{f}$ is the intensity of the electric field of the source, $E_{S}$ is the intensity of the electric field in the shield-protected area

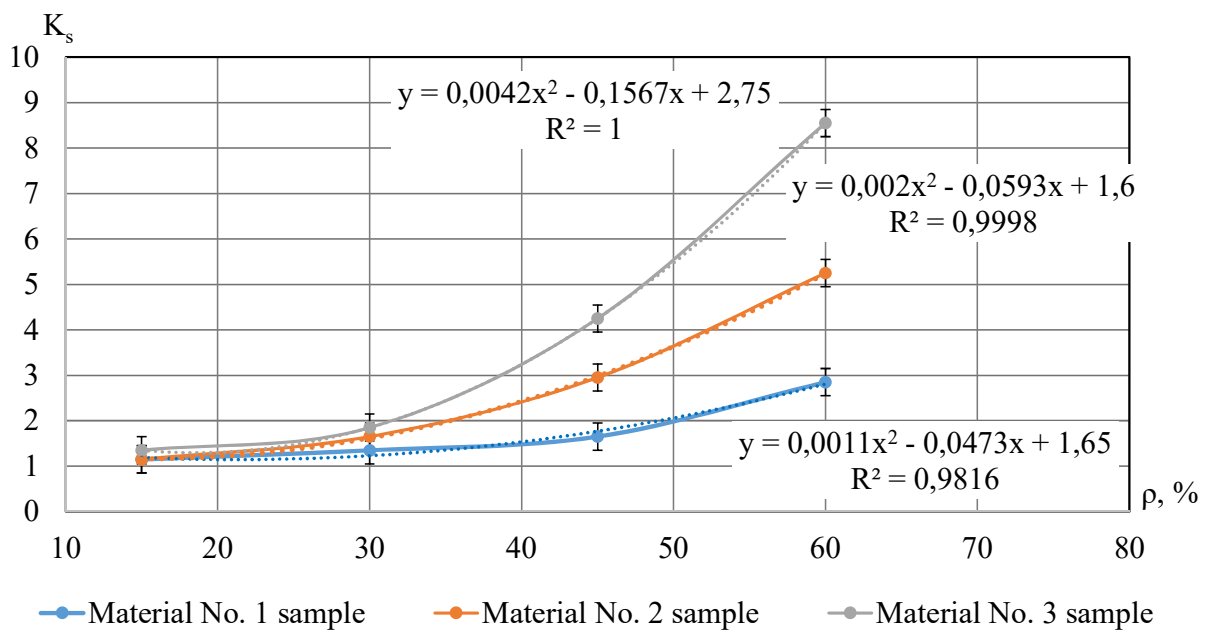

Fig. 1. Dependence of the shielding coefficients $K_{S}$ of the electric field of industrial frequency on the content of the screening substance 
The results of measuring the shielding coefficients of the magnetic field of the industrial frequency with induction of 280-285 $\mu$ T are given in Table 2 and shown in Fig. 2. the undesirable direction). The reflection coefficients measured by the procedure described in [12] are given in Table 4 and shown in Fig. 4.

Table 2

Shielding coefficients $K_{S}$ of the electric field of industrial frequency

\begin{tabular}{|c|c|c|c|c|}
\hline \multirow{2}{*}{$\begin{array}{c}\text { Sample of } \\
\text { material }\end{array}$} & $15 \%$ & $30 \%$ & $45 \%$ & $60 \%$ \\
\cline { 2 - 5 } & $1.2-1.3$ & $1.5-1.6$ & $2.5-2.6$ & $3.7-3.8$ \\
\hline No. 1 & $1.4-1.5$ & $1.9-2.0$ & $3.8-3.9$ & $7.7-7.8$ \\
\hline No. 2 & $1.2-1.3$ & $1.6-1.7$ & $2.8-2.9$ & $5.6-5.7$ \\
\hline No. 3 & \multicolumn{4}{|c|}{$K_{S}$} \\
\hline
\end{tabular}

Shielding coefficients $K_{S}$ of the electric field of ultra-high frequency

\begin{tabular}{|c|c|c|c|c|}
\hline \multirow{2}{*}{$\begin{array}{c}\text { Sample of } \\
\text { material }\end{array}$} & \multicolumn{4}{|c|}{$K_{S}$} \\
\cline { 2 - 5 } & $15 \%$ & $30 \%$ & $45 \%$ & $60 \%$ \\
\hline No. 1 & $1.2-1.3$ & $1.3-1.4$ & $1.8-1.9$ & $4.0-4.1$ \\
\hline No. 2 & $1.3-1.4$ & $1.6-1.7$ & $2.9-3.0$ & $5.5-5.6$ \\
\hline No. 3 & $1.7-1.8$ & $2.3-2.4$ & $4.0-4.1$ & $7.8-7.9$ \\
\hline
\end{tabular}

$\mathrm{K}_{\mathrm{s}}$

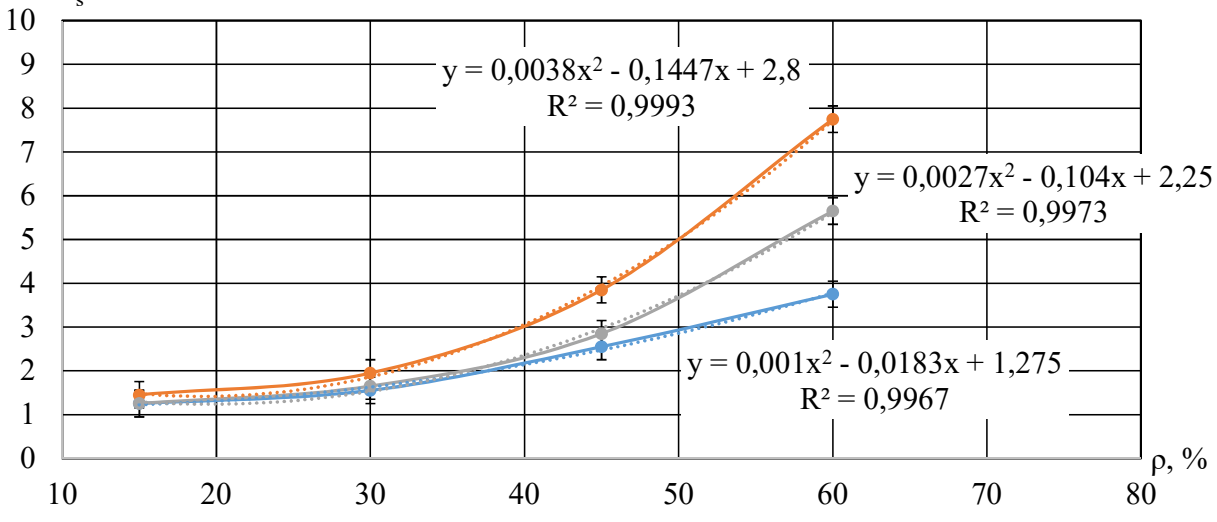

$\rightarrow-$ Material No. 1 sample $\quad \rightarrow$ Material No. 2 sample $\quad \rightarrow$ Material No. 3 sample

The resulting correlation coefficients indicate the reliability of our findings. The statistical treatment of experimental data has made it possible to derive analytical functions of the dependence of a shielding coefficient on the content of the screening material. These functions in the process of designing the protective material make it possible to uniquely determine the content of the screen filler in the dielectric matrix to obtain the required shielding coefficient of both low-frequency and high-frequency electromagnetic fields.

Our results indicate that the developed materials are suitable for protecting people from elec-

The results of measuring the shielding coefficients of the electromagnetic field with a frequency of $2.45 \mathrm{GHz}$ with an energy flow density of $178-182 \mu \mathrm{W} / \mathrm{cm}^{2}$ are given in Table 3 and shown in Fig. 3.

For high-frequency radiation, it is important to contribute protection by reflecting electromagnetic waves into the overall shielding coefficient (the possibility of reflection in tromagnetic influences under industrial and household conditions, at least with a screen substance content above $45 \%$ (by weight). The content of fillers by volume is much smaller due to significant differences in the density of metal-containing filler and paints used. Therefore, the filler does not significantly affect the adhesion of paint to the surface, which is important for the practical use of the obtained protective materials.

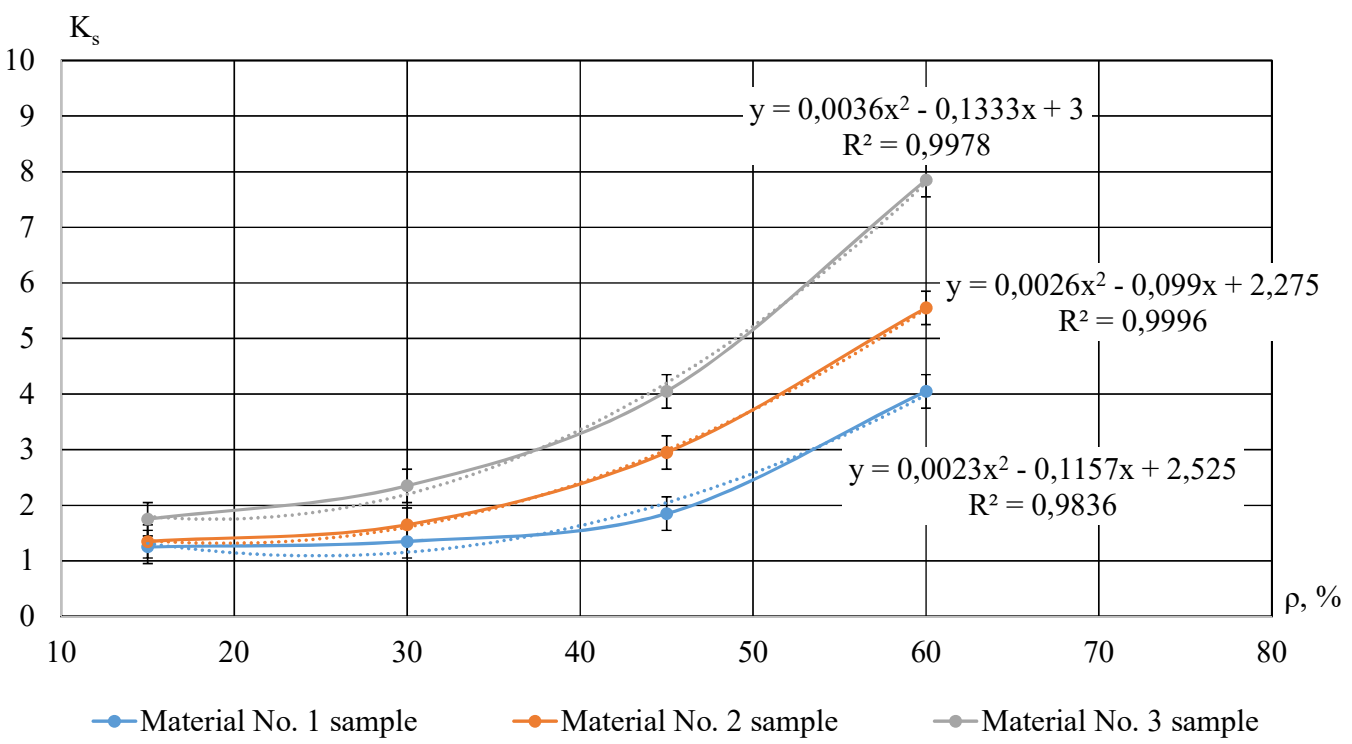

Fig. 3. Dependence of the shielding coefficients $K_{S}$ of the electric field of ultra-high frequency on the content of the screening substance 
Table 4 where $V_{m}, V_{d}$ are the filler and dielectric volumes; $\rho_{m}, \rho_{d}$ are

Shielding coefficients $K_{r}$ of the electric field of ultra-high frequency *

\begin{tabular}{|c|c|c|c|c|}
\hline \multirow{2}{*}{$\begin{array}{c}\text { Sample of } \\
\text { material }\end{array}$} & \multicolumn{4}{|c|}{$K_{r}$} \\
\cline { 2 - 5 } & $15 \%$ & $30 \%$ & $45 \%$ & $60 \%$ \\
\hline No. 1 & - & - & $0.10-0.15$ & $0.22-0.23$ \\
\hline No. 2 & - & - & $0.15-0.18$ & $0.28-0.29$ \\
\hline No. 3 & - & - & $0.22-0.24$ & $0.32-0.34$ \\
\hline
\end{tabular}

Note: *Reflection effect for the filler concentrations of $15,30 \%$ is beyond the sensitivity of the measuring device

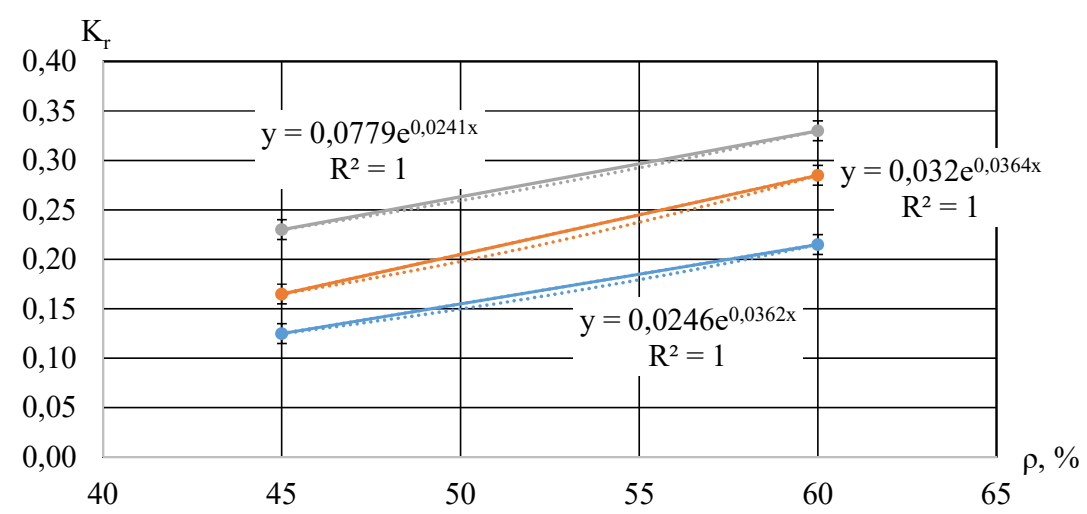

$\rightarrow$ Material No. 1 sample $\rightarrow-$ Material No. 2 sample $\rightarrow-$ Material No. 3 sample

Fig. 4. Dependence of the shielding coefficients $K_{r}$ of the electric field of ultrahigh frequency on the content of the screening substance the filler and dielectric density; $k=\frac{P_{m}}{P_{d}}$ is the ratio of filler
and dielectric weight.

To determine the effective dielectric permeability of the composition $\varepsilon$, it is advisable to use the Maxwell-Garnet ratio, which holds for $v_{m}<1 / 3$ :

$$
\frac{\varepsilon-\varepsilon_{d}}{\varepsilon+2 \varepsilon_{d}}=v_{m} \frac{\varepsilon_{m}-\varepsilon_{d}}{\varepsilon_{m}+2 \varepsilon_{d}}
$$

$\varepsilon_{d}, \varepsilon_{m}$ are the dielectric permeability of the matrix and filler, $v_{m}$ is the volumetric share of the filler in a dielectric matrix.

The materials of the matrix and filler are common, so their dielectric permeability is known. If necessary, these values could be measured by standard devices.

In many cases, filler particles cannot be considered spherical, so, to determine the effective dielectric permeability of the composition, it is advisable to use the Odelevsky formula:

$$
\varepsilon=\varepsilon_{d}\left[1+\frac{v_{m}\left(\varepsilon_{m}-\varepsilon_{d}\right)}{\left(1-\frac{v_{m}}{v_{k}}\right) F\left(\varepsilon_{m}-\varepsilon_{d}\right)+\varepsilon_{d}}\right]
$$

5. 2. Devising the principles of designing protective materials by predicting the magnetic and electrophysical properties of materials

For calculations on the design of protective structures of the required efficiency, quantitative values of magnetic and electrophysical characteristics of materials are needed such as effective magnetic and dielectric permeability, as well as specific conductivity. However, these data are not available for composite materials. Therefore, it is advisable to calculate them based on the parameters of the composition components and the ratio of their content in the material.

Assume the filler particles are the same in size and shape and are distributed evenly into a dielectric matrix. We believe that the volumetric coefficient of filling the matrix with shielding particles is small, that is, the particles do not affect each other. In this case, one can use a Lorentz ratio for the magnetic permeability of magnetodielectrics:

$$
\mu=1+\frac{v_{m}\left(\mu_{m}-1\right)}{1+\frac{1-v_{m}}{3}\left(\mu_{m}-1\right)},
$$

where $\mu$ is the effective magnetic permeability of the magnetodielectric, $\mu_{m}$ is the magnetic permeability of the filler, $v_{m}$ is the volume content of the filler in the material.

In most cases, in the manufacture of a composition, it is convenient to change the weight content of the filler (including this work), which is due to its powdery state and high dispersion.

For recalculation, one should use the following ratio:

$$
\frac{V_{m}}{V_{d}}=\frac{k \rho_{d}}{\rho_{m}}
$$

where $\varepsilon_{d}, \varepsilon_{m}$ are the dielectric permeability of the matrix and filler, $v_{m}$ is the volume content of the filler, $v_{k}$ is the critical volume content of the filler at which the shielding particles are in contact with each other, $F$ is the depolarization coefficient

$$
F=\ln \left(\frac{2 l}{d}\right) /\left(\frac{l}{d}\right)^{2}
$$

where $l$ is the average particle length, $d$ is the average diameter.

$$
v_{k}=\frac{[3 F(1-F)]^{0,6}}{[4 F \sqrt{l / d}+(1+F) / \sqrt{l / d}]^{0,6}} .
$$

The $v_{k}$ indicator is very important for the design of composite materials. As shown in [10], a sharp increase in the shielding coefficient is observed when the threshold of electric current leakage is achieved (conductivity circuits are formed by contact of filler particles). This is consistent with the positions of the electrodynamics of solid media.

By calculating the effective values of magnetic and dielectric permeability, one could determine all the parameters that are necessary for the design of the protective material. Based on the following ratio

$$
\varepsilon=\varepsilon^{\prime}+\varepsilon^{\prime \prime}
$$

and considering that in the ultra-low and low-frequency range, the imaginary part of the complex dielectric permeability $\varepsilon^{\prime \prime}$ is much larger than $\varepsilon^{\prime}$ and could be neglected

$$
\varepsilon=\varepsilon^{\prime \prime}=\frac{4 \pi \sigma}{\omega},
$$


where $\sigma$ is the specific conductivity of the material, $\omega$ is the circular frequency of the electromagnetic field.

This ratio makes it possible to determine the specific conductivity of the material. Based on the obtained data, it is possible to determine the equivalent depth of field penetration into the material (the depth at which the field is reduced by $e=2.7$ times).

For example, for electromagnetic fields of ultra-high and higher frequencies, the equivalent penetration depth $\delta$ is defined as:

$$
\delta=\sqrt{\frac{2}{\omega \mu_{a} \sigma}},
$$

where $\mu_{a}$ is the absolute magnetic permeability, $\mu_{a}=\mu_{0} \mu$.

If, for example, the field needs to be reduced by 10 times, the thickness of the protective screen should not be less than $2.3 \delta$.

\section{Discussion of results of studying the protective properties of composite materials and determining ways to improve them}

The analysis of the measurement results given in Tables $1-3$ reveals that the general shielding coefficients for the filler content of $45 \%$ and $60 \%$ (by weight) are quite acceptable for the practical application of the developed mixtures. The lowest shielding coefficients of the material based on a water-dispersed paint could be explained by partial oxidation of the filler in the presence of water. This effect is not manifested at such concentrations of iron ore concentrate in a geopolymer paint. It is known that the protective properties largely depend on the conductivity of the material. Therefore, in the presence of contact between individual particles, the conductivity in mixtures No. 2 and No. 3 is greater due to the lack of oxidation and a greater conductivity of the GreyX material compared to ore concentrate. In addition, its dispersion is higher, which simplifies the formation of conductivity chains.

The recalculation of the concentrations of the filler by weight into volumetric concentration according to the ratios given in section 6 indicates that the weight concentrations of $45 \%$ and $60 \%$ correspond to the volumetric concentrations of $15 \%$ and $28 \%$. At such concentrations, the conductivity threshold is reached and there is a significant increase in shielding coefficients.

To design protective materials, information about the magnetic and electrophysical properties of materials at certain volume concentrations of filler is required. The calculated values of the relative magnetic permeability of materials are 1.27-1.48. These data were compared to the results of direct measurements of relative magnetic permeability according to the procedure described in [13]. For example, for mixture No. 2, the relative magnetic permeability is $1.50-1.51$, which is acceptable to match the calculated value (1.48).

The calculated relative dielectric permeability values are $3.42-3.65$, and the measured permeability values are 3.45-3.68. For example, for mixture No. 3, the relative dielectric permeability is maximum. The above predetermines the largest shielding factor of the magnetic component of the electromagnetic field of industrial frequency by material No. 2 and the electrical component - by material No. 3. At the same time, the calculated specific conductivity values are an order of $10^{-3} \mathrm{~S} / \mathrm{m}$, which is enough to ensure acceptable shielding coefficients. Increasing the conductivity of materials leads to an undesirable increase in reflection coefficients. This coefficient depends on the ratios of wave resistances $Z$ of the distribution environment of electromagnetic waves (air) and protective material, which are determined from the following ratio:

$$
Z=\sqrt{\frac{\mu_{a}}{\varepsilon_{a}}},
$$

where $\mu_{a}$ is the absolute magnetic permeability of the materi$\mathrm{al}, \varepsilon_{a}$ is the absolute dielectric permeability (60\%).

For material No. 1, the wave resistance is $249 \Omega$.

Determination of the reflection coefficient is carried out by a known ratio:

$$
K_{S}=\frac{Z_{1}-Z_{2}}{Z_{1}+Z_{2}}
$$

where $Z_{1}$ and $Z_{2}$ are the wave resistances of the propagation medium of electromagnetic waves and the protective material.

Given that the air wave resistance is $377 \Omega$, the reflection coefficient of the material $K_{S}=0.2$, which acceptably coincides with the experimental data given in Table 4 .

The above indicates that in the presence of data on the electromagnetic situation under specific conditions and the necessary degrees of shielding (comparison with the maximum permissible values), a preliminary calculation assessment of the composition of the screen mixture for the manufacture of a protective screen is possible.

The study reported here has certain limitations. Measurement of total shielding coefficients and the reflection coefficient of electromagnetic waves was performed for typical frequencies and limited concentrations of the shielding substance in the dielectric matrix.

The caveat of this study is the lack of data in reference sources on the magnetic and electrophysical properties of composite materials. However, in the future, this disadvantage could be eliminated by creating a reference database on the electrophysical properties of composite materials. Determining the effectiveness of the protective material, depending on the conditions of its use, is important for the introduction of the automated design of protective composite materials. To automate the design processes of protective materials and structures of the required efficiency, it is advisable to perform a series of experimental and theoretical studies. Such research is due to the need to create a database on the actual values and frequency dependences of effective magnetic and dielectric permeability and their specific conductivities on the composition and physical characteristics of the components of composite materials.

\section{Conclusions}

1. For the manufacture of liquid compositions for shielding electrical, magnetic, and electromagnetic fields of the most common frequencies, it is advisable to use standard paints with the addition of iron ore concentrate and pigment metal-containing additives as a screening substance. The content of the screening substance up to $60 \%$ (by weight) for the used fillers corresponds to the content by volume up to $28 \%$ and does not significantly affect the adhesion of 
the mixture to the surfaces on which composite materials are applied. The total shielding coefficients of the electromagnetic field of industrial frequency of mixture layers $0.22-0.25 \mathrm{~mm}$ thick with the content of the shielding substance of $15-60 \%$ (by weight) for a water-dispersed paint are 1.1-2.9; for a geopolymer paint $-1.1-5.3$. Adding $50 \%$ of GreyX pigment metallic substance to the mixture increases shielding coefficients to 8.6. Magnetic field shielding coefficients also assume acceptable values. The shielding coefficients of ultra-high frequency electromagnetic field are up to 7.9. Low shielding coefficients of the material based on a water-dispersed paint could be explained by the oxidation of the metal filler and a decrease in the electrical conductivity of the material. In the presence of a filler up to $60 \%$, the reflection coefficient of an ultra-high frequency electromagnetic field does not exceed 0.23 for a water-dispersed paint and 0.34 for a geopolymer paint. This makes it possible to choose the formulation of the composition with the desired protective properties.

2. It has been shown that forecasting of protective properties of materials is possible by calculating the magnetic and electrophysical properties of the material. Our assumptions (point particles are evenly distributed in the body of the matrix) do not introduce a significant error to the result of relative calculations. The calculated values of magnetic permeability for the selected material are 1.48; measured, 1.50-1.51; for the relative dielectric permeability, the calculated values are 3.65 , measured -3.65 . At the same time, the coefficient of reflection of ultramagnetic waves of ultra-high frequency is estimated at 0.20 , and that measured experimentally $-0.22-0.23$. This indicates the possibility to estimate determining the effectiveness of the protective material depending on the conditions of its use and to implement the automated design of protective composite materials.

\section{References}

1. Vergallo, C., Dini, L. (2018). Comparative Analysis of Biological Effects Induced on Different Cell Types by Magnetic Fields with Magnetic Flux Densities in the Range of 1-60 mT and Frequencies up to $50 \mathrm{~Hz}$. Sustainability, 10 (8), 2776. doi: https:// doi.org/10.3390/su10082776

2. Duhaini, I. (2016). The effects of electromagnetic fields on human health. Physica Medica, 32, 213. doi: https://doi.org/10.1016/ j.ejmp.2016.07.720

3. Patil, N., Velhal, N. B., Pawar, R., Puri, V. (2015). Electric, magnetic and high frequency properties of screen printed ferrite-ferroelectric composite thick films on alumina substrate. Microelectronics International, 32 (1), 25-31. doi: https:// doi.org/10.1108/mi-12-2013-0080

4. Mondal, S., Ganguly, S., Das, P., Khastgir, D., Das, N. C. (2017). Low percolation threshold and electromagnetic shielding effectiveness of nano-structured carbon based ethylene methyl acrylate nanocomposites. Composites Part B: Engineering, 119, 41-56. doi: https://doi.org/10.1016/j.compositesb.2017.03.022

5. Yadav, R. S., Kuritka, I., Vilcakova, J., Machovsky, M., Skoda, D., Urbanek, P. et. al. (2019). Polypropylene Nanocomposite Filled with Spinel Ferrite $\mathrm{NiFe}_{2} \mathrm{O}_{4}$ Nanoparticles and In-Situ Thermally-Reduced Graphene Oxide for Electromagnetic Interference Shielding Application. Nanomaterials, 9 (4), 621. doi: https://doi.org/10.3390/nano9040621

6. Tahmasebi Birgani, M. J., Zabihzadeh, M., Aliakbari, S., Behrouz, M. A., Hosseini, S. M. (2019). Evaluation of Putty Metal for Internal Shielding for Patient Protection in Electron Therapy by Monte Carlo Study. Jundishapur Journal of Natural Pharmaceutical Products, 14 (2), e12589. doi: https://doi.org/10.5812/jjnpp.12589

7. Zhang, L., Bi, S., Liu, M. (2020). Lightweight Electromagnetic Interference Shielding Materials and Their Mechanisms. Electromagnetic Materials and Devices. doi: https://doi.org/10.5772/intechopen.82270

8. Belyaev, A. A., Bespalova, E. E., Lepeshkin, V. V. (2015). Radio absorbing materials based on finishing construction materials for protection against microwave radiation of base stations of cellular communication. Trudy VIAM, 6, 80-88.

9. Barsukov, V., Senyk, I., Kryukova, O., Butenko, O. (2018). Composite Carbon-Polymer Materials for Electromagnetic Radiation Shielding. Materials Today: Proceedings, 5 (8), 15909-15914. doi: https://doi.org/10.1016/j.matpr.2018.06.063

10. Senyk, I. V., Kuryptya, Y. A., Barsukov, V. Z., Butenko, O. O., Khomenko, V. G. (2020). Development and Application of Thin Wide-Band Screening Composite Materials. Physics and Chemistry of Solid State, 21 (4), 771-778. doi: https://doi.org/10.15330/ pcss.21.4.771-778

11. Guzii, S., Kryvenko, P., Guzii, O., Yushkevich, S. (2019). Determining the effect of the composition of an aluminosilicate binder on the rheotechnological properties of adhesives for wood. Eastern-European Journal of Enterprise Technologies, 6 (6 (102)), 30-38. doi: https://doi.org/10.15587/1729-4061.2019.185728

12. Glyva, V., Podkopaev, S., Levchenko, L., Karaieva, N., Nikolaiev, K., Tykhenko, O. et. al. (2018). Design and study of protective properties of electromagnetic screens based on iron ore dust. Eastern-European Journal of Enterprise Technologies, 1 (5 (91)), 10-17. doi: https://doi.org/10.15587/1729-4061.2018.123622

13. Radionov, A. V., Podoltsev, A. D., Radionova, A. A. (2017). Express - method for determining the quality of a magnetic fluid for operation in the working gap of a magnetic fluid seal. IOP Conference Series: Materials Science and Engineering, 233, 012038. doi: https://doi.org/10.1088/1757-899x/233/1/012038 